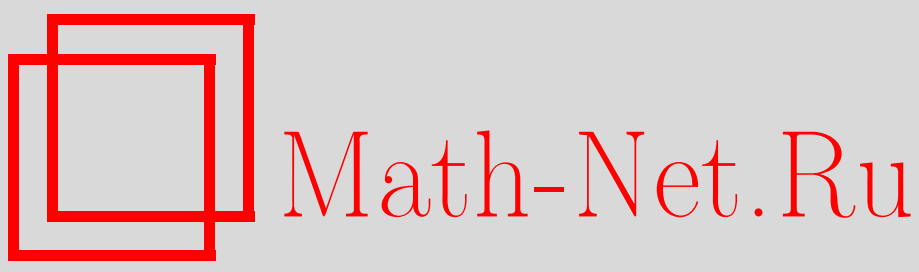

А. Я. Белов, Размерность Гельфанда-Кириллова относительно свободных ассоциативных алгебр, Матем. сб., 2004, том 195, номер 12, 3-26

DOI: https://doi.org/10.4213/sm862

Использование Общероссийского математического портала Math-Net.Ru подразумевает, что вы прочитали и согласны с пользовательским соглашением http://www . mathnet.ru/rus/agreement

Параметры загрузки:

IP: 54.198 .187 .58

26 апреля 2023 г., 08:13:50 
УДК $512.552 .4+512.554 .32+512.664 .2$

\title{
А.Я. Белов
}

\section{Размерность Гельфанда-Кириллова относительно свободных ассоциативных алгебр}

\begin{abstract}
В работе вычисляется размерность Гельфанда-Кириллова $\operatorname{GKdim}(A)$ относительно свободной ассоциативной алгебры $A$ над произвольньгм основным полем. Эта размерность определяется сложснстным типом алгебры $A$ или набором полупрямых произведений матричных алгебр над кольцом многочленов, входящих в многообразие $\operatorname{Var}(A)$. Доказательство сравнительно элементарно и не использует локальной представимости относительно свободных алгебр.

Библиографиия: 15 названий.
\end{abstract}

\section{§. Введение}

Пусть $A$ - алгебра, $a_{1}, \ldots, a_{s}$ - ее образуюшие. Все кольца и алгебры в настоящей статье считаются конечно порожденньми (сокращенно к.п.). Алгебра $A$ линейно представима множеством $M$, если $M$ порождает $A$ как векторное пространство. Функиия роста $V_{A}(n)$ алгебры $A$ определяется как размерность пространства, натянутого на слова длины не вьше $n$; производяшая функция $\sum V_{A}(n) t^{n}$ называется рядом Гильберта алгебры $A$. Для абсолютно свободной $s$-порожденной алгебры $A$ имеет место равенство $V_{A}(n)=s^{n}$ и, значит,

$$
H_{A}(T)=\frac{1}{1-s \cdot T} .
$$

Поэтому ряды Гильберта абсолютно свободных алгебр рациональны. Рациональность рядов Гильберта для относительно свободных алгебр установлена автором (см. [1], [2]). Для конечномерной алгебры функция роста начиная с некоторого $n$ становится постоянной, и в этом случае ряд Гильберта рационален.

Функция роста зависит от выбора системы образуюших. Но если ввести отношение эквивалентности на множестве функций $f \equiv g \Leftrightarrow \exists c \in \mathbb{N}: \forall n f(c n) \geqslant g(n)$ и $g(c n) \geqslant f(n)$, то класс эквивалентности $V_{A}(n)$ уже есть инвариант самой алгебры. Размерностью Гельфанда-Кириллова алгебры $A$ называют предел $\operatorname{GKdim}(A)=$ $\lim _{n \rightarrow \infty}\left(\ln V_{A}(n) / \ln n\right)$, если таковой существует (а существует не всегда, см. [3]); $\operatorname{GKdim~есть~инвариант~самой~алгебры.~Если~} A$ коммутативна, то $\operatorname{GKdim}(A)$ есть степень ее трансцендентности.

Известно, что $\operatorname{GK} \operatorname{dim}(A)$ может принимать значения $\{0,1,2, \infty\}$, а также любое число из луча $[2, \infty)$, причем если

$$
\underline{\operatorname{GKdim}(A)}=\varliminf_{n \rightarrow \infty} \frac{\ln V_{A}(n)}{\ln n}=0 \text { или } 1,
$$


то и $\overline{\operatorname{GKdim}(A)}$ тоже равна 0 или 1 . Равенство $\operatorname{GKdim}(A)=0$ равносильно конечномерности алгебры $A$. Для коммутативного кольца $R$ размерность $\operatorname{GKdim}(R)$ всегда определена и равна его степени трансцендентности. Эта размерность была введена Гельфандом и Кирилловым. Они показали, что для $U(L)$ - универсальной обертывающей алгебры $n$-мерной алгебры Ли $L$ - имеет место равенство $\operatorname{GK} \operatorname{dim}(U(L))=n$. В. А. Уфнаровский показал, что если $L-$ алгебра Ли и

$$
\operatorname{GKdim}(L)=r>0,
$$

то $V_{A}(n)$ растет как $\exp \left(n^{1-1 / r}\right)$. (И с помощью этого результата построил пример конечно определенной алгебры промежуточного роста.)

Напомним, что алгебра $A$ называется PI-алгеброй, если в ней выполняется некоторое нетривиальное тождество. Это значит, что многочлен вида

$$
f\left(x_{1}, \ldots, x_{m}\right)=\sum_{\sigma \in S_{m}} \alpha_{\sigma} x_{\sigma(1)} \cdots x_{\sigma(m)}
$$

(коэффициенты $\alpha_{\sigma} \in \mathbb{F}$ - не все нули) обрашается в нуль при подстановке любых элементов алгебра $A$ вместо переменных $x_{i}$.

Множество многочленов, тождественно обращающихся в нуль на алгебре, замкнуто относительно операции подстановки многочлена вместо переменных и, кроме того, образует идеал в кольце (некоммутативных) многочленов. Такой идеал называется $T$-идеалом. Известно, что множество всех $T$-идеалов - это множество идеалов, устойчивых относительно всех эндоморфизмов. Класс алгебр, в котором выполняется некоторый набор тождеств, называется многообразием, а свободньй объект в этом многообразии называется относительно свободной алгеброй.

Размерность Гельфанда-Кириллова вычислялась для некоторых относительно свободных алгебр: для алгебры обших матриц см. [4], для свободной алгебры из многообразия $\operatorname{Var}(\mathbb{O})$, порожденного алгеброй Кэли-Диксона $\mathbb{O}$, а также простой исключительной йордановой алгебры $H C_{3}$ в случае, когда $\operatorname{Char}(\mathbb{K}) \neq 2,3$, это сделано в работах [5], [6]. В работах [7], [8] вычислялась размерность Гельфанда-Кириллова для алгебры общих матриц, а также некоторых других относительно свободных алгебр, в частности, многообразия, порожденного алгеброй Кэли-Диксона $\mathbb{O}$. В этих же работах исследовались некоторые близкие асимптотики.

Основная теорема настоящей работы устанавливает размерность ГельфандаКириллова для относительно свободных ассоциативных алгебр.

Теорема 0.1. Пусть $A-s$-порожденная относительно свободная алгебра. Тогда $\operatorname{GK\operatorname {dim}}(A)$ зависит только от ее сложностного типа. А именно, она равна максимальной размерности Гельфанда-Кириллова полупрямого произведения ${ }^{1}$ алгебр общих матрич из $\operatorname{Var}(A)$. Размерность Гельфанда-Кириллова такого полупрямого произведения равна сумме размерностей Гельфанда-Кириллова сомножителей. Сложностные типы у алгебры $А$ и подалгебры, порожденной двумя ее образующими, совпадают.

\footnotetext{
${ }^{1}$ Полупрямые произведения определены в конце $\S 1$.
} 
Размерность Гельфанда-Кириллова свободной s-порожденной алгебры $A$ из $\operatorname{Var}\left(\mathbb{M}_{n_{1}} \rtimes \cdots \rtimes \mathbb{M}_{n_{k}}\right)$ равна

$$
k+(s-1) \sum_{i=1}^{k} n_{i}^{2} .
$$

Аналогичньй результат для экспонент в ряде коразмерностей получен в [9]. Было установлено, что минимальные многообразия алгебр (над полем нулевой характеристики) с данной экспонентой ${ }^{2}$ суть полупрямые произведения $T$-первичных многообразий (частным случаем $T$-первичного многообразия служит многообразие, порожденное алгеброй общих матриц). Экспонента такого многообразия есть сумма экспонент сомножителей. (То же верно и для размерности Гельфанда-Кириллова.) Тем самым экспонента многообразия также определяется его сложностньм типом (случай конечного базисного ранга см. в [10], общий случай анонсирован в [11], [12], полный текст выйдет в Adv. Math.). Экспоненты всех $T$-первичных многообразий в нулевой характеристике вычислены в работах A. Giambruno, М.В. Зайцева, С.П. Мишенко, A. Regev'a. Применительно к полупрямьм произведениям матричных алгебр результаты упомянутых авторов означают, что $e(\mathfrak{M})=\sum_{i=1}^{k} n_{i}^{2}$, что похоже на равенство (1). Мы также используем технику, разработанную указанными авторами (см. теорему 3.2 ).

$\mathrm{B}$ частном случае, когда $k=1$, получается п. а) следующей теоремы, которой мы будем пользоваться.

Теорема 0.2. а) Размерность Гельфанда-Кириллова для s-порожденной алгебры общих матрич порядка п равна $1+(s-1) n^{2}$.

б) Указанная размерность равна степени трансцендентности центра.

Этот результат был впервые установлен К. Прочези [4]; А.В. Гришин [7], [8] дал несколько другое доказательство этого утверждения, установив равенство размерности Гельфанда-Кириллова алгебры общих матриц и ее идеала, порожденного центральными многочленами, и размерности Гельфанда-Кириллова алгебры общих матриц со следом. Аналогичные результаты были получены для многообразия, порожденного алгеброй Кэли-Диксона.

Прокомментируем теорему 0.2. Пусть $A_{1}, \ldots, A_{s}$ - общие матрицы, $A$ - алгебра, ими порожденная, $s \geqslant 2$. Выбрав подходящий базис, матрицу $A_{1}$ можно считать диагональной. Кроме того, можно добиться того, что непосредственно надглавной диагональю матрицы $A_{2}$ (рядом с ней) будет стоять диагональ из $n-1$ единицы (в этом месте рассуждений требуется наличие второй матрицы, т.е. неравенство $s \geqslant 2)$. Тогда легко убедиться в том, что в случае общего положения дальнейшее приведение невозможно и избавиться от оставшихся $n+\left(n^{2}-(n-1)\right)+n^{2}(s-2)=$ $n^{2}(s-1)+1$ свободных параметров нельзя. Алгебра общих матриц вкладывается в алгебру матриц над кольцом многочленов от $n^{2}(s-1)+1$ переменных. Отсюда получается верхняя оценка: $\operatorname{GKdim}(A) \leqslant n^{2}(s-1)+1$.

Верхняя оценка в общем случае дает следующее

\footnotetext{
${ }^{2}$ Если размерность пространства, порожденного полилинейньми словами длины $n$ от образующих $x_{1}, \ldots, x_{n}$ в свободной алгебре из $\mathfrak{M}$, порожденной множеством $\left\{x_{i}\right\}_{i=1}^{\infty}$, ассимптотически эквивалентна $e^{n}$, то число $e=e(\mathfrak{M})$ называется экспонентой многообразия $\mathfrak{M}$.
} 
ПрЕДЛОЖЕНИЕ 0.3. Пусть $A-$ представимая алгебра сигнатуры $\Omega$ с образующими $a_{1}, \ldots, a_{s}, \quad\left\{e_{i}\right\}-$ базис векторного пространства с операторами, в которое она вложсена. Пусть далее $a_{j}=\sum_{i} \lambda_{i j} e_{i}, j=1, \ldots, s$, коэффициенты $\lambda_{i}$ порохдают ассоциативно-коммутативное кольцо $R, A$ вложена в $R$-алгебру, являюшуюся нётеровым $R$-модулем. Тогда $\operatorname{GKdim}(A)$ не превосходит степени трансцендентности кольца $R$.

В этой связи хотелось бы поставить

Вопрос. Верно ли, что минимальная размерность кольца представления $R$ для представимой ассочиативной алгебры А равна ее размерности Гельфанда-Кириллова?

Неравенство $\operatorname{dim}(R) \geqslant G \operatorname{Gim}(A)$ очевидно. Попытки получить обратную оценку были связаны с диофантовыми проблемами, аналогичными тем, что наблюдаются при изучении рядов Гильберта представимых алгебр [3]. ${ }^{3}$ Рассмотрим произведение матриц над кольцом многочленов $C A^{n} D$. Коэффициенты при матричных единицах имеют вид:

$$
\sum_{i=1}^{k} P_{i}(n) \lambda_{i}^{n}
$$

где $k$ - размер матриц, $\lambda_{i}$ - собственные числа $A$ (из некоторого конечного алгебраического расширения кольца представления), $P_{i}$ - многочлены степени не выше $k$.

Чтобы показать, что для представимой алгебры размерности Гельфанда-Кириллова 1 можно обеспечить размерность кольца представления 1, надо показать, что если размерность основного кольца 2 или больше, то пространство, порожденное элементами вида (2), пересекается по нулю с некоторым нетривиальньм идеалом. Пока это сделать не удалось. Аналогичным образом, если размерность Гельфанда-Кириллова равна $s$, то она совпадает с сушественной высотой, и надо рассматривать множество произведений вида

$$
C_{0} U_{1}^{n_{1}} C_{1} \cdots C_{s-1} U_{s}^{n_{s}} C_{s},
$$

а также пространства $S_{\alpha}$, порожденные коэффициентами при матричных единицах:

$$
\sum_{I} P_{i}\left(n_{1}, \ldots, n_{s}\right) \lambda_{i_{1}}^{n_{1}} \cdots \lambda_{i_{s}}^{n_{s}},
$$

где $I$ пробегает некоторое конечное множество.

И чтобы показать, что для представимой алгебры размерности Гельфанда-Кириллова $s$ можно обеспечить размерность кольца представления $s$, надо показать, что если размерность основного кольца равна $s+1$, то пространство, порожденное элементами вида (2), пересекается по нулю с некоторым нетривиальным идеалом.

Мы начнем с изучения полупрямых произведений и их применения к вычислению размерности Гельфанда-Кириллова относительно свободных представимых

\footnotetext{
${ }^{3}$ Удалось установить только, что размерность Гельфанда-Кириллова представимой алгебры произвольной сигнатуры есть целое число и совпадает с существенной высотой (см. [3]).
} 
алгебр. Затем относительно свободный ассоциативный случай будет сведен к представимому (этот этап можно опустить, воспользовавшись представимостью конечно порожденных относительно свободных алгебр, но лучше действовать более элементарно).

Сведение относительно свободного случая к представимому осуществляется с помощью техники представимых пространств, являюшихся расширениями нильпотентами некоторой представимой алгебры. Далее надо установить, что расширение нильпотентным идеалом относительно свободной представимой ассоциативной алгебры $A$ в многообразии $\operatorname{Var}(A)$ имеет ту же размерность Гельфанда-Кириллова, что и исходная алгебра.

По всей видимости, впервые свободные радикальные расширения рассматривал (и широко эксплуатировал при исследовании проблемы конечной базируемости) А.В. Гришин. Он показал следуюшее [7], [8]. Дано многообразие $\mathfrak{M}$, порожденное конечномерной ассоциативной алгеброй. Рассматривается расширение $($ в $\mathfrak{M}) d$-порожденной относительно свободной алгебры из $\mathfrak{M}$ свободньми элементами $\left\{x_{i}\right\}$ и рассматриваются слова, в которые дополнительные переменные входят не более $c$ раз. Тогда при достаточно большом $c$ размерность Гельфанда-Кириллова пространства $S_{c}$, порожденного этими словами, стабилизируется, и эта стабилильная величина, именуемая показателем роста, вычисляется. (Она совпадает, как мы сейчас видим, с размерностью Гельфанда-Кириллова относительно свободных алгебр.)

Введение дополнительных радикальных переменных в работах [7], [8] (восходящим к работам Ж. Левина) обеспечивает контроль за радикальной частью в $S_{c}$ (т.е. позволяет обойти эффекты наличия возможных соотношений между радикальной и полупростой частью, связывающей первичные компоненты). По сути дела одной из наших целей является преодоление указанных трудностей в обшей ситуации, что позволяет перейти от вычисления стабильных величин для расширений нильпотентами к самой размерности исходной алгебры.

Для обеспечения контроля над радикалом в общей ситуации нужен дополнительный ход - рассмотрение замыканий (по Зарискому, см. [1], или конструкцией ограниченно свободных расшшрений, см. конец 11 и и контроль над базисным рангом. Более конструктивньй способ действий, выбранньй в этой статье, - paбота с экстремальньм $T$-идеалом в полупрямых произведениях с использованием обобщения теоремы М.В. Зайцева и A. Giambruno для произвольных полей (теорема 3.2 ).

Автор выражает благодарность руководителям и участникам семинара "теория колец" В. Н. Латьшеву, А. В. Михалеву, А. В. Гришину, В. Т. Маркову, а также Б.Р. Френкину за помошь в работе.

\section{§1. Вспомогательные сведения}

1.1. Представимые алгебры. Алгебра называется алгеброй нётерового mипа, если она является алгеброй и одновременно нётеровьм модулем над нётеровым ассоциативно-коммутативным кольцом $R$. Алгебра называется представимой, если она вкладывается в алгебру нётерового типа. При этом кольцо $R$ называется кольцом представления, и можно считать, что $R$ содержит единицу. Известно, что кольцо представления любой к.п. представимой алгебры над 
нётеровым ассоциативно-коммутативным кольцом $\Phi$ может быть выбрано нётеровым. Все ассоциативно-коммутативные кольца, встречающиеся в настоящей работе, если не оговорено противное, будут считаться нётеровыми. Поскольку любое нётерово кольцо является целым конечным расширением кольца многочленов, то любая представимая к.п. алгебра представима матрицами над кольцом многочленов (для алгебр над произвольным ассоциативно-коммутативным кольцом дело обстоит сложнее). Кроме того, любая представимая алгебра вкладывается в алгебру, являющуюся свободньм модулем над кольцом представления.

ОПРЕДЕЛЕнИЕ 1.1. Назовем идеал I представимым, если факторпо нему есть представимая алгебра. Идеал тождеств представим, если он является $T$-идеалом представимой алгебры, или (что равносильно) относительно свободная алгебра из соответствующего многообразия представима. Минимальное $n$ такое, что алгебра $A$ вкладывается в алгебру, являющуюся модулем ранга $n$ над своим центром, называется порядком представления. Если идеал $I$ представим, то порядок представления $I$ есть порядок представления $A / I$.

ОПРЕДЕЛЕНИЕ 1.2. Пусть $B$ - ассоциативная алгебра, $\left\{\beta_{i}\right\}$ - ее образующие, $\left\{b_{i}\right\}_{i \in I}$-множество всех ее элементов, $\left\{\delta_{i j}\right\}_{i \in I, j=1, \ldots, m}$ - набор независимых коммутативных переменных. Каноническое алгебрачческое представление порядка т есть алгебра

$$
\widehat{B}^{(m)}=B\left[\delta_{i j}\right] / \operatorname{id}\left(\left\{b_{i}^{m}+\delta_{i 1} b_{i}^{m-1}+\cdots+\delta_{i m}\right\}_{i \in I}\right) .
$$

Если индекс $i$ будет пробегать множество, отвечающее словам степени не выше $s$ от образуюших алгебры $B$, то получившийся объект мы будем называть каноническим алгебраическим представлением длины $s$ порядка $m$ и обозначать $\widehat{B}^{(m, s)}$.

Пусть $b \in B$. Расширим алгебру $B$ свободными коммутативньми константами $\delta_{i}, i=1, \ldots, n-1$, и рассмотрим фактор по идеалу id $\left(b^{m}+\delta_{1} b^{m-1}+\cdots+\delta_{m}\right)$. Алгебра $B$ естественным образом отображается в эту алгебру, а ядро этого отображения называется препятствием $к$ алгебраичности порядка $m$ әлемен$m a b$. Ядро при каноническом алгебраическом представлении порядка $m$ называется препятствием $к$ алгебраичности порядка $m$. Аналогично определяется препятствие к алгебраичности порядка т системъ әлементов $\left\{b_{i}\right\}$.

Пусть система форм $\delta_{i}(b)$ удовлетворяет тождеству Гамильтона-Кэли. Это значит, что в алгебревыполняется тождество $a^{n}+\delta_{1}(a) a^{n-1}+\cdots+\delta_{n}(a) \equiv 0$. Тогда естественно строится каноническое представление Гамильтона-Кәли поряджа $n$, а соответствуюшее ядро называется препятствием $\kappa$ тождеству Гамильтона-Кәли порядка п.

Говоря неформально, если все элементы алгебры $B$ (соответственно слова длины не выше $s$ ) "насильно" с делаем алгебраическими степени $m$, то получим каноническое алгебраическое представление степени $m$ (соответственно длины $s$ ).

Аналогичньм образом определяется идеал $J_{k}$ - npensтствие $\kappa$ nредставимости матрицами порядка $k$ над нётеровым кольцом.

Из локальной конечномерности алгебраических алгебр и алгебраичности представимых алгебр вытекает следуюшая

ЛЕмма 1.3 (о каноническом представлении). а) Каноническое алгебраическое представление является нётеровым модулем над значениями оператора 
следа. То же верно для представления длинь $s$, если $s$ не превосходит $m$ или $\mathrm{PIdeg}(B)$.

б) Если В представима матрицами порядка $m$, то естественнье отображения $B \mapsto \widehat{B}^{(m)}, B \mapsto \widehat{B}^{(m, s)}$ являются вложениями.

в) Если В представима, т.е. вкладьвается в алгебру нётерового типа, то каноническое алгебраическое представление некоторого порядка является вложением.

Пусть $M-$ нётеров модуль над нётеровым коммутативным кольцом $S$. Тогда конечная сумма $\bigoplus_{i} \bigotimes^{k_{i}} M$ тоже нётерова. Пусть $A-$ представимая алгебра, вложенная в алгебру нётерового типа $\widehat{A}, \sqcup=\left\{t_{i}\right\}$-конечный набор букв. Рассмотрим множество слов $\mathscr{U}_{k}$, в которые буквы из $\sqcup$ входят не более чем $k$ раз. Рассмотрим далее всевозможные специализации элементов алгебры $A$ в $\mathscr{U}_{k}$.

ПреДЛОЖЕНИЕ 1.4. Пусть $A-$ представимая алгебра. Тогда существует такой конечный набор $\mathscr{M}$ әлементов из $A$, что для любого многочлена $F(\vec{y}, \vec{\Lambda})$, имеющего степень однородности не выше $k$ относительно переменных из конечного набора $\vec{\Lambda}$, тождественное равенство $F(\vec{y}, \vec{\Lambda})$ нулю равносильно равенству нулю $F(\vec{y}, \vec{\xi})$ для любых $\vec{\xi} \in \mathscr{M}^{k}$.

Это предложение можно переформулировать на языке расширений ограниченно свободньпи элементами порядка $k: n$-элементный набор элементов $\mathscr{M}=\left\{m_{i}\right\} \subset A$ называется свободным порядка $k$, если для любого многочлена $F$ степени не выше $k$ относительно переменных из $\left\{t_{i}\right\}=\Lambda$ тождественное равенство $F(\vec{y}, \vec{\Lambda})=0$ равносильно обрашению в нуль результатов подстановок $m_{i} \mapsto t_{i}$.

Рассмотрим расширение $A_{\operatorname{Var}(A)}\langle\Lambda\rangle$ и всевозможные специализации $t_{i} \mapsto m_{i}$, $i=1, \ldots,|\Lambda|$, каждая из которых отвечает гомоморфизму этого расширения на алгебру $A$. Поскольку набор $\mathscr{M}$ конечен, возникает гомоморфизм расширения $A_{\operatorname{Var}(A)}\langle\Lambda\rangle$ в прямую сумму $\bigoplus^{k} A, k$ - число всех таких специализаций. Элементы $A$ вложатся как константы, образы элементов из $\Lambda$ будут свободными элементами порядка $k$ в образе. Получилось свободное расширение порядка $k$ с помощъю набора $\mathscr{M}$ свободного порядка $k$.

1.2. Следы и формы. Относительно свободная алгебра, как правило, алгеброй нётерового типа не является. Однако если ее расширить значениями оператора следа (в положительной характеристике - также операторов форм), то расширенная алгебра уже будет иметь нётеров тип. Цель построения экстремального идеала обычно в том, что экстремальный идеал $I$ оказывается замкнутым относительно умножения на некоторое подкольцо кольца форм. Он совпадает с экстремальным идеалом расширенной алгебры.

Основное соображение, принадлежащее Ю.П. Размыслову, таково. Пусть $f(\vec{x}, \vec{y})$ - многочлен, полилинейный и кососимметричный по $\vec{x}=\left(x_{1}, \ldots, x_{n}\right)$, $V$ - векторное пространство, порожденное $x_{i}$, оператор $A \in \operatorname{End}(V)$. Тогда $f(A \cdot \vec{x}, \vec{y})=f\left(A x_{1}, \ldots, A x_{n}, \vec{y}\right)=\operatorname{det}(A) f(\vec{x}, \vec{y})$. Положим $A=E+t a$. Имеет место разложение по степеням $t$ :

$$
f(A \cdot \vec{x}, \vec{y})=\left(\sum_{k=0}^{n} \Phi_{k}(a) t^{k}\right) f(\vec{x}, \vec{y})
$$


где $\Phi_{k}(a)$ есть форма $k$-го порядка от оператора $a$. Она равна следу оператора $\bigwedge^{k}(a)$, действуюшего на векторном пространстве $\bigwedge^{k}(V)$. Этот след равен сумме главных миноров порядка $k$ матрицы оператора $a$. В частности, $\Phi_{1}(a)=\operatorname{Tr}(a)$, $\Phi_{n}(a)=\operatorname{det}(a), \Phi_{0}(a)=1$. Если $A=E+\sum t_{i} a_{i}$, то

$$
\operatorname{det}(A)=\sum_{\vec{k}} t_{1}^{k_{1}} \cdots t_{s}^{k_{s}} \Phi_{k_{1}, \ldots, k_{s}}\left(a_{i_{1}}, \ldots, a_{i_{s}}\right),
$$

где $s$ - число координат вектора $\vec{k}$ (разное при разных параметрах суммирования $\vec{k}$ ), форма $\Phi_{k_{1}, \ldots, k_{s}}$ есть частичная линеаризация формы $\Phi_{\sum} k_{i}$.

Величина $\Phi_{k_{1}, \ldots, k_{s}}$ равна сумме главных миноров порядка $k=\sum k_{i}$, у которых $k_{1}$ столбцов взяты от матрицы $a_{1}, k_{2}$ столбцов - от $a_{2}$ и т.д. Сумма берется также по всем способам выбора столбцов. Подробнее тождества с формами (т.е. набором дополнительных операций, однородных относительно входящих в них переменных) см. в [13].

Легко видеть, что $\widehat{\Phi}_{1}(a)=n \operatorname{Tr}(a), \widehat{\Phi}_{n^{2}}(a)=\operatorname{det}(a)^{n}$. Поэтому

$$
n \operatorname{Tr}(Z) C\left(x_{1}, \ldots, x_{n^{2}} ; y_{1}, \ldots, y_{n^{2}}\right)=\left.\sum_{i=1}^{n^{2}} C\left(x_{1}, \ldots, x_{n^{2}} ; y_{1}, \ldots, y_{n^{2}}\right)\right|_{x_{i}=Z x_{i}} .
$$

Переменные из набора $\vec{y}$ называются прокладками.

Известно, что любой элемент в алгебре общих матриц цел над значениями операторов форм (в положительной характеристике нельзя обойтись следами, например, все следы степеней скалярной матрицы порядка $p$ равны нулю, см. [13]).

Суммируем полученные результаты, а также несколько других классических фактов, которые нам понадобятся.

ТЕоРема 1.5 (Ю.П. Размыслов). а) В алгебре общих матрии размера $n$ выполняется тождество Капелли порядка $n^{2}+1$ и не выполняется тождество Капелли порядка $n^{2}$.

б) Имеют место равенства $\left(x_{i}\right.$ альтернируются, $y_{i}-$ “прокладки"):

$$
\begin{aligned}
& \operatorname{det}(Z) C\left(x_{1}, \ldots, x_{n^{2}} ; y_{1}, \ldots, y_{n^{2}}\right)=C\left(Z x_{1}, \ldots, Z x_{n^{2}} ; y_{1}, \ldots, y_{n^{2}}\right) \\
& =\left.C\left(x_{1}, \ldots, x_{n^{2}} ; y_{1}, \ldots, y_{n^{2}}\right)\right|_{x_{i}=Z x_{i} \forall i}, \\
& \operatorname{Tr}\left(Z_{1}\right) \operatorname{Tr}\left(Z_{2}\right) C\left(x_{1}, \ldots, x_{n^{2}} ; y_{1}, \ldots, y_{n^{2}}\right) \\
& =\left.\sum_{i=1}^{n^{2}} C\left(x_{1}, \ldots, x_{n^{2}} ; y_{1}, \ldots, y_{n^{2}}\right)\right|_{x_{i}=Z_{1} x_{i} Z_{2}} .
\end{aligned}
$$

(Равенство (6) полезно для работы в положительной характеристике.)

Полином называется центральным, если он не является тождеством и его значения лежат в центре алгебры. Как показал Ю.П. Размыслов, в алгебре общих матриц $n$-го порядка всегда существует ненулевой центральный полином, обращающийся в нуль на матрицах менњшего порядка. Этот полином устойчив относительно умножения на следы (формы) и будет обозначаться через $Z_{n}$ (его конструкция различается в случае нулевой и положительной характеристик). Устойчивость означает, что в результате умножения на след (форму) получается элемент алгебры общих матриц, а не расширенной алгебры.

Из теоремы Ширшова о высоте (если все слова длины не выше степени алгебры алгебраичны, то такая алгебра конечномерна) непосредственно вытекает 
ПрЕДЛОЖЕНИЕ 1.6. а) Пусть $Y$ - множество слов длины не въше $2 n$ алгебры общих матрич $M_{n}, Z$ есть множсество форм от значений әлементов из $Y$. Тогда расширение $M_{n}[Z]$ алгебры $M_{n}$ чело над $\mathbb{K}[Z]$ и является нётеровым модулем над $\mathbb{K}[Z]$.

Аналогичное утверждение справедливо для любой представимой алгебры А сложности п. Расиирение $\widehat{A}$ представимой алгебры А значением оператора следа на әлементах описанного выше типа является нётеровым модулем над коммутативным кольцом. (Следь (форми) определяются исходя из представления.)

Сложность алгебры $A$ есть максимальное $n$ такое, что $\mathbb{M}_{n} \in \operatorname{Var}(A)$.

1.3. Диаграммы Кемера и внутренние следы. С каждой диаграммой Юнга $D$ можно связать множество непересекающихся наборов переменных $\left\{\Lambda_{i}\right\}$, соответствующих ее столбцам. При этом число элементов в наборе $\Lambda_{i}$ равно длине соответствуюшего столбца. Через $S(D)$ обозначим $T$-идеал, порожденный многочленами, полилинейными и кососимметричньми по каждому $\Lambda_{i}$.

Определим величину $b(\mathfrak{M})$ как наибольшее натуральное $b$, удовлетворяюшее следуюшему условию:

существуют диаграммы $D$ из сколь угодно большого числа клеток такие, что все столбиь имеют длину не меньше $b$ и при этом не все полиномы из $S(D)$ являются тождествами многообразия $\mathfrak{M}$.

Для алгебры $C$ положим $b(C)=b(\operatorname{Var}(C))$. Если $C$ нильпотентна, то $b(C)=0$, а если $C$ порождает многообразие всех ассоциативных алгебр, то $b(C)=\infty$.

ОПРЕДЕЛЕНИЯ. Пусть $\mathfrak{M}$ - многообразие алгебр, $D$ - диаграмма Юнга, все столбцы которой имеют длину не меньше $b=b(\mathfrak{M})$. Если кроме того $\left.S(D)\right|_{\mathfrak{M}} \neq 0$, то такую диаграмму мы будем называть любопьтной. Диаграмма $D$ называется интересной, если сушествуют сколь угодно большие любопытные диаграммы, содержащие $D$. Назовем столбец любопытной диаграммы $D$ большим, если его длина строго больше $b$, а если она равна $b$, то он мальий. Мальцм является набор переменных, отвечающих малому столбцу, а больиим - большому. Множество больших столбцов образует голову $H(D)$ диаграммы $D$. Диаграмма назьвается әкстремальной, если она интересная и, кроме того, при $H\left(D^{\prime}\right) \supset H(D)$ и $D^{\prime} \supset D$ все многочлены из $S\left(D^{\prime}\right)$ являются тождествами $\mathfrak{M} ; k(H)$ есть минимальное число малых столбцов в экстремальной диаграмме с головой $H$ (если такой диаграммы нет, то $k(H)=\infty$ ). Хорошей диаграммой с головой $H$ назьвается экстремальная диаграмма не менее чем с $k+1$ малым столбцом. Экстремальная диаграмма называется диаграммой Кемера, если все большие столбцы имеют длину $b+1$. Тогда $d$ обозначает их число, а $k$-минимальное количество малых столбцов в диаграмме Кемера. Итак, минимальная диаграмма Кемера описывается параметрами $b, d$ и $k$. Тем самым для многообразия $\mathfrak{M}$ определены величины $b(\mathfrak{M}), d(\mathfrak{M})$ и $k(\mathfrak{M})$. Типы многообразий или тройки $(b, d, k)$ сравниваются так: $\left(b_{1}, d_{1}, k_{1}\right) \prec\left(b_{2}, d_{2}, k_{2}\right)$, если выполняется одно из трех условий:

i) $b_{1}<b_{2}$;

ii) $b_{1}=b_{2}, d_{1}<d_{2}$;

iii) $b_{1}=b_{2}, d_{1}=d_{2}, k_{1}>k_{2}$. 
Промежутки между переменными, соответствующими диаграмме, называются прокладками. Когда это не вызывает недоразумений, термин “прокладка" будет использоваться и при изучении значений соответствующих многочленов (если подстановки переменных из набора $\bigcup \Lambda_{i}$ фиксированы). В дальнейшем, если не оговорено обратное, под диаграммой Кемера понимается хороиая диаграмма Кемера.

Операторы форм можно определить внутренним образом. Пусть в алгебре выполнено любое тождество вида $S_{\Lambda_{1}} \cdots S_{\Lambda_{k+1}} f=0$, где $\left|\Lambda_{i}\right|=m+1$ при всех $i$, $S_{\Lambda}$ обозначает альтернирование по переменньм из набора $\Lambda$.

Пусть полилинейный многочлен $g$ кососимметричен по наборам переменных из $\Lambda_{i}$, где $\left|\Lambda_{i}\right|=n+1$ при $i>0$ и $\left|\Lambda_{0}\right|=\left|\Lambda_{0}^{\prime}\right|=n$. Можно считать, что $g$ имеет вид: $g=S_{\Lambda_{0}} S_{\Lambda_{0}^{\prime}} S_{\Lambda_{1}} \cdots S_{\Lambda_{k}} f$. Для каждого $x$ определим оператор внутренней формы $\delta_{k}(x)$ (линейный относительно $g$ ) на Т $(g)$ следующим образом:

$$
\delta_{k}(x)=\left.\sum_{I \subset\{1, \ldots n\},|I|=k} g\right|_{a_{i}=x a_{i} \forall i \in I, a_{i} \in \Lambda_{0}} .
$$

Полная линеаризация этого оператора есть оператор $\Psi\left(x_{1}, \ldots, x_{k}\right)$, который выражается равенством

$$
\Psi\left(x_{1}, \ldots, x_{k}\right)=\left.\sum_{i_{1}<\cdots<i_{k}} g\right|_{a_{i_{\alpha}}=x a_{i_{\alpha}}, a_{i_{\alpha}} \in \Lambda_{0}}
$$

В частности, определен линейньй по $x$ оператор внутреннего следа $\operatorname{Tr}(x)=$ $\delta_{1}(x)$ :

$$
\operatorname{Tr}(x) g=\left.\sum_{i=1}^{k} g\right|_{a_{i}=x a_{i}, a_{i} \in \Lambda_{0}}
$$

Следуюшее предложение является непосредственньм переносом основной теоремы работы [14] на экстремальные многочлены (см. [1]).

ПРЕДЛОЖЕНИЕ 1.7. Пусть $g \in S(D), g=S_{\Lambda_{0}} S_{\Lambda_{0}^{\prime}} S_{\Lambda_{1}} \cdots S_{\Lambda_{d}}(f), D-\partial u a-$ грамма Кемера, $\left|\Lambda_{0}\right|=\left|\Lambda_{0}^{\prime}\right|=m,\left|\Lambda_{i}\right|=m+1, i=1, \ldots, d=d(A)$. Тогда справедливь следующие утверәсдения.

а) Операторы внутренних форм, определенные выше, коммутируют. В частности, $\operatorname{Tr}(x) \operatorname{Tr}(y)=\operatorname{Tr}(y) \operatorname{Tr}(x)$.

б) Выполняется равенство $\operatorname{Tr}(a b)=\operatorname{Tr}(b a)$.

в) Пусть многочлен $g$ линеен по переменной $z \notin \bigcup \Lambda_{i}$. Положим $g_{h}=\left.g\right|_{h \mapsto z}$. Пусть $h^{(k)}=h^{\prime} a^{k} h^{\prime \prime}, g^{(k)}=g_{h^{(k)}}$.

Тогда имеет место равенство ("тождество Гамильтона-Кәли"):

$$
g^{(n)}=\sum_{k=1}^{n} \delta_{k}(a) g^{(n-k)}
$$

г) Пусть $h$ принадлежст препятствию $к$ тождеству Гамильтона-Кәли порядка п. Тогда $g_{h}=0$. 
д) Подпространство $\mathrm{T}(\mathrm{g})$, кососимметрическое по наборам переменных из $\Lambda_{i}$, является нётеровом модулем над операторами $\delta_{k}$ и вкладывается в представимую алгебру.

е) Пусть полилинейный многочлен $g$ линеен по переменной $z \notin \bigcup \Lambda_{i}$, также полилинеен и кососимметричен по наборам переменных из $\Lambda_{i}$, где $\left|\Lambda_{i}\right|=n+1$ при $i>0,\left|\Lambda_{0}\right|=n$ (в отличие от ситуации в остальньх пунктах, набор $\Lambda_{0}^{\prime}$ отсутствует).

Тогда имеет место аналог тожсества Гамильтона-Кәли:

$$
g^{(n)}=\sum_{k=1}^{n} \delta_{k}(a) g^{(n-k)}
$$

Если $h$ принадлежст препятствию к каноническому алгебраическому представлению порядка $\mathrm{m}$, то $g_{h}=0$.

ж) Пространство многочленов, полилинейных по переменным из набора

$$
\Lambda=\Lambda_{0} \cup \Lambda_{0}^{\prime} \cup \bigcup_{i>0} \Lambda_{i}
$$

и кососимметричных относительно $\Lambda_{0}, \Lambda_{0}^{\prime}$ икаждого $\Lambda_{i}, i>0$, представимо. Естественный морфизм в каноническое алгебраическое представление некоторого порядка является вложением.

ЗАМЕчАнИЕ. В работе [14] утверждения предложения 1.7 сформулированы и доказаны для случая, когда наборы $\Lambda_{i}, i>0$, отсутствуют. Рассматривались алгебры, в которых выполнялась система $C_{n+1}$ и определялись внутренние следы для многочленов, кососимметрических относительно набора из $n$ переменных (или двух наборов из $n$ переменных $\Lambda_{0}$ и $\Lambda_{0}^{\prime}$ ). Поскольку наша ситуация отличается только наличием дополнительных наборов $\Lambda_{i}, i>0$, которые в вычислениях не участвуют, все выкладки проходят дословно (см. [1]).

1.4. Представимые пространства и тестовые алгебры. Предложение 1.7 можно переформулировать на языке тестовых (представимых) пространств.

ОПреДЕлЕНИЕ 1.8. Пространство $S \subseteq A$ назьвается представимым, если оно пересекается по нулю с препятствием к представимости некоторого порядка. Назовем пространство $S \subset B$ представимым в алгебре $B$, если имеется морфизм $B$ в алгебру нётерового типа, ограничение которого на $S$ является вложением.

Для диаграммы $D$ многообразия $\mathfrak{M} \supseteq \operatorname{Var}(A)$ и алгебры $A$ определим подпространство $S_{D, \mathfrak{M}}(A) \subseteq A_{\mathfrak{M}}\left\langle\left\{\Lambda_{i}\right\}\right\rangle$ как пространство многочленов, полилинейных и кососимметричных по переменньм из каждого набора $\Lambda_{i}$, соответствующего диаграмме $D$ (свободное произведение берется в многообразии $\mathfrak{M})$. Когда ясно, какая алгебра имеется в виду, мы будем писать просто $S_{D, \mathfrak{M}}$.

Поскольку в вычислениях, устанавливающих предложение 1.7, участвуют переменные только из одного набора $\Lambda_{i}$, доказательство естественного аналога предложения 1.7 переносится на случай нескольких наборов. Имеем следующее 
ПРЕДЛОЖЕНИЕ 1.9. Пусть $D$ - әкстремальная диаграмма для многообразия $\mathfrak{M} \supseteq \operatorname{Var}(A), S=S_{D, \mathfrak{M}}$. Тогда если $D$ содержит мальй столбеи, то пространство $S$ представимо.

Сопоставим диаграмме $D$ систему наборов переменных $\xi_{i j} \in \Lambda_{i}$, где $\Lambda_{i}$ - набор сильно антикоммутирующих переменных, отвечающий $j$-му столбцу диаграммы $D$.

Пусть $\mathfrak{M}$ - многообразие алгебр, $D$ - диаграмма Кемера. Рассмотрим алгебpy $K_{0, \mathfrak{M}}(A, D)=A *_{\mathfrak{M}}\left\langle\left\{\xi_{i j}\right\}\right\rangle / Q$, где идеал $Q$ порожден мономами $M_{\alpha}$, содержащими два вхождения любой из переменных $\xi_{i j}$, а также элементами вида $M\left(\xi_{i j_{1}}, \xi_{i j_{2}}\right)-M\left(\xi_{i j_{2}}, \xi_{i j_{1}}\right)$. Алгебра $K_{0, \mathfrak{M}}(A, D)$ есть расширение $A$ в многообразии $\mathfrak{M}$ системой сильно антикоммутирующих переменных, заданной диаграммой $D$. Обычно $\mathfrak{M}=\operatorname{Var}(A)$, и это подразумевается, если индекс $\mathfrak{M}$ опушен. В любом случае, для корректности конструкций необходимо, чтобы выполнялось включение $\mathfrak{M} \supseteq \operatorname{Var}(A)$. Это всегда подразумевается.

Пусть $J_{b+1}$ есть идеал в $K_{0, \mathfrak{M}}(A, D)$, порожденный значениями полиномов вида $f\left(u_{1}, \ldots, u_{b+1}, \vec{y}\right)$, где $u_{k}$ - мономы от образуюших $A, f$ полилинейно и кососимметрично относительно $\left\{u_{i}\right\}$. Положим $K_{1, \mathfrak{M}}(A, D)=K_{0, \mathfrak{M}}(A, D) / J_{b+1}$, $K_{\mathfrak{M}}(A, D)$ есть пространство (идеал) в $K_{1, \mathfrak{M}}(A, D)$, порожденное мономами, полилинейными по всем переменным вида $\xi_{i j}$. В силу экстремальности $D$ соответствуюшие пространства в алгебрах $K_{1, \mathfrak{M}}(A, D)$ и $K_{0, \mathfrak{M}}(A, D)$ естественньм образом изоморфны.

В пространстве $K_{\mathfrak{M}}(A, D)$ естественным образом определено умножение на внутренние следы (формы), и это действие задает структуру нётерова модуля на пространстве $K_{\mathfrak{M}}(A, D)$.

ПреДЛОЖЕНИЕ 1.10 (о представимых пространствах). Пусть $D$ - әкстремальная диаграмма. Тогда справедливы следующие утверждения.

а) Соответствие $A \mapsto K_{\mathfrak{M}}(A, D)$ есть ковариантный функтор. Пространство $K_{\mathfrak{M}}(A, D)$ всегда представимо и, кроме того, $K_{\mathfrak{M}}(A, D)$ изоморфно пространству $K_{\mathfrak{M}}\left(A^{\prime}, D\right)$ для некоторого $A^{\prime}$ - фактора $A$ по некоторому представимому идеалу. При этом $T$-идеал $H(D)$ алгебры $K_{1, \mathfrak{M}}(A, D)$ лежит в пространстве $K_{\mathfrak{M}}(A, D)$.

б) Выполняются равенства $\operatorname{Var}(A)=\operatorname{Var}\left(K_{0, \mathfrak{M}}(A, D)\right)=\operatorname{Var}\left(K_{1, \mathfrak{M}}(A, D)\right)$.

в) Пространства $S_{1}(A, D)$ и $S_{0}(A, D)$ в алгебрах $K_{1, \mathfrak{M}}(A, D)$ и $K_{0, \mathfrak{M}}(A, D)$ соответственно, порожденные мономами, полилинейными по всем переменным вида $\xi_{i j}$, естественныцм образом изоморфны (иногда обозначаются $S(A, D))$, и әти пространства представимы.

Если характеристика основного поля равна нулю, то пространство $K_{\mathfrak{M}}(A, D)$ изоморфно подпространству $A_{\mathfrak{M}}\langle X\rangle$-пространству многочленов с коэффициентами в $A$, полилинейных и кососимметричных относительно соответствующих наборов переменных.

По представимому пространству $S_{\Lambda}$ можно определить представимую алгебру $A(D)$, которая получается из канонического (операторного) алгебраического представления $A$ путем расширения переменными из набора $\Lambda$ и взятия фактора по мономам, содержашим две одинаковые переменные из $\Lambda$.

1.5. Сложностной тип и полупрямые произведения. Полупрямым произведением алгебр $A$ и $B$ (обозначение $A \rtimes B$ ) назьвается факторалгебра алгебры 
$A+A * B+B$ по идеалу, порожденному элементами вида $b * a, b \in B, a \in A$. Иногда полупрямое произведение называют ниль-произведением.

Легко видеть, что полупрямое произведение раскладывается в сумму своих подалгебр, нумеруюшихся парами индексов $(j, p)$ и соответствуюших пространствам, порожденным элементами вида $E_{i j} t E_{p q}$. Каждая такая алгебра изоморфна алгебре "блочных верхнетреугольных" матрищ и порождает то же многообразие, что и любая такая алгебра.

Аналогичньм образом полупрямое произведение нескольких матричных алгебр размера $n_{i}$ изоморфно сумме своих подалгебр, которые мы обозначаем $U\left(\mathbb{F}, d_{1}, \ldots, d_{m}\right)$. Каждая такая подалгебра задается матрицами вида

$$
\left(\begin{array}{cccc}
\mathbb{M}_{n_{1}} & * & \cdots & * \\
0 & \mathbb{M}_{n_{2}} & * & \vdots \\
\vdots & & \ddots & * \\
0 & \ldots & & \mathbb{M}_{n_{k}}
\end{array}\right)
$$

Поэтому в дальнейшем в этой работе под полупрямым произведением алгебр матриц будем понимать подалгебру вида $U\left(\mathbb{F}, d_{1}, \ldots, d_{m}\right)$.

Блоки $\mathbb{M}_{n_{i}}$ называются клетками.

Хорошо известна теорема Левина, утверждаюшая, что идеал тождеств полупрямого произведения есть произведение идеалов тождеств сомножителей.

Сложностныц типом алгебры $A$ мы будем называть класс всех полупрямых произведений матричных алгебр $M_{1} \rtimes \cdots \rtimes M_{k}$ из $\operatorname{Var}(A)$. Это понятие включает в себя классическую сложность $\operatorname{PIdeg}(A)$ (максимальный размер матриц из $\operatorname{Var}(A))$ и сложность Латышева (максимальный размер верхнетреугольных матриц из $\operatorname{Var}(A))$. Число разнотипных клеток в минимальном графе представления - параметр $b(A)$ Кемера - есть максимальная размерность алгебры из сложностного типа.

ОПРеДЕЛЕНИЕ 1.11. Пусть $B$ - конечномерная алгебра, $\left\{\vec{e}_{i}\right\}_{i=1}^{n}-$ ее базис, $\left\{x_{k l}\right\}_{k=1 l=1}^{s}-$ независимые переменные, $s$-порожсденной алгеброй общих әлементов из $B$ называется алгебра, порожденная образуюшими $a_{i} \sum_{l=1}^{n} x_{i l} \vec{e}_{l}$.

Это определение непосредственно переносится на многоосновные алгебры произвольной сигнатуры. Алгебра общих элементов относительно свободна и порождает однородное многообразие.

\section{§ 2. Относительно свободные ассоциативные алгебры}

2.1. Верхние оценки. Пусть $n_{1}, \ldots, n_{k}$-натуральные числа, $A$ есть алгебра обших элементов для полупрямого произведения $\mathbb{M}_{n_{1}} \rtimes \cdots \rtimes \mathbb{M}_{n_{k}} c s$ образуюшими, причем $s \geqslant 2$ (см. определение (1.11)). В этом случае путем суммирования чисел параметров, относящихся к клеткам, получается такая оценка:

$$
\operatorname{GKdim}(A) \leqslant k+(s-1) \sum_{i \in I} n_{i}^{2} .
$$

При этом суммирование идет по множеству индексов $I$, отвечающих матричньм алгебрам над бесконечньм центром. (Таким образом, клетки конечного типа 
не влияют на значение $\operatorname{GKdim}(A)$ и в качестве сложностного типа берется максимальный набор из клеток бесконечного типа.)

В силу теоремы 3.2 для алгебры сложностного типа $\left(n_{1}, \ldots, n_{k}\right)$ получается такая же оценка. Отметим, что радикальные параметры, относящиеся к матрицам $A_{i}$, входят во все произведения в ограниченной степени. А число мономов ограниченной степени конечно. Поэтому они не оказывают влияния на верхнюю оценку. (Но от них зависит коэффициент при старшем члене в выражении для функции роста.) Впрочем для верхней оценки достаточно получить ее для алгебры $\widehat{A}$, и она получается как максимум из сумм, относящихся к клеткам связной компоненты графа. При этом в качестве кольца представления можно взять кольцо многочленов от описанных вьше свободных параметров.

ЗАмечАниЕ. Величина $\sum n_{i}^{2}$ равна размерности полупростой части $b(a)$ в обозначениях А.Р. Кемера. Она совпадает с величиной $b(A)$, определенной в $\S 1$.

Теперь рассмотрим многообразие $\mathfrak{M}$, порожденное полупрямым произведением алгебр матриц над конечным полем и алгебр матриц с бесконечным центром.

Относительно свободная алгебра $A$ из $\mathfrak{M}$ представима блочно диагональными матрицами, у которых в блоках, отвечающих множителям с бесконечным центром, стоят различные независимые переменные (своя переменная для каждой позиции и для каждой образующей) из кольца многочленов. Блокам, отвечающим множителям конечного типа, тоже отвечают различные переменные, но только "свободные переменные для $i$-го блока" берутся из кольца $R_{i}=\mathbb{F}\left[\vec{x}_{i}\right] / K_{i}$, где $K_{i}$ есть $T$-идеал, порожденный элементами вида $y^{q_{i}}-y$. При этом $q_{i}=p^{k_{i}}$ есть минимальное натуральное число такое, что в центре $i$-го множителя вьполняется тождество $y^{q_{i}}-y \equiv 0$. Кольцо $R_{i}$ конечно порождено (число образуюших $R_{i}$ равно произведению $n_{i}^{2}$ и числа образующих $A$ ) и конечно. Поэтому множители конечного типа на верхнюю оценку влияния не оказывают.

$\mathrm{C}$ другой стороны, рассмотрим полупрямое произведение $A^{\prime}$ клеток бесконечного типа в том же порядке (пропустив клетки конечного типа). В силу теоремы $3.2 A^{\prime}$ вкладьвается в $A$, и из результатов п. 2.2 следует, что $\mathfrak{M}^{\prime}=\operatorname{Var}\left(A^{\prime}\right)$ есть максимальное подмногообразие $\mathfrak{M}$, порожденное полупрямым произведением алгебр матриц с бесконечным центром. И размерность Гельфанда-Кириллова относительно свободной $s$-порожденной алгебры $A$ равна максимальной размерности однородной $s$-порожденной алгебры из $\operatorname{Var}(A)$.

2.2. Нижние оценки. Полупрямое произведение алгебр матрищ представимо матрицами, вдоль главной диагонали которых стоят клетки - блоки фиксированных размеров, над ними - что угодно, под ними - нули. Ограничение на клетку дает эпиморфизм на алгебру матриц. Такую алгебру мы называем полупрямым произведением матричных алгебр. Выбирая общие элементы, получаем алгебру общих блочно-треугольных матриц. Мы говорим, что элемент (множитель) содержит (данную) клетку (радикал), если он принадлежит идеалу, порожденному соответствующими матричными единицами.

Отметим, что размерность Гельфанда-Кириллова $s$-порожденной относительно свободной алгебры из многообразия $\mathfrak{M}$ равна максимальной размерности Гельфанда-Кириллова $s$-порожденной алгебры из $\mathfrak{M}$, являюшейся полупрямым произведением алгебр обших матриц (в нашей терминологии). Набор таких алгебр из $\mathfrak{M}$ служит сложностной характеристикой многообразия, по которой ведется ин- 
дукция. Эквивалентное определение сложностного типа: это множество наборов типов клеток ( “составов путей” ), которые может содержать одновременно элемент алгебры.

Итак, пусть $A$ есть полупрямое произведение $\mathbb{M}_{1} \rtimes \cdots \rtimes \mathbb{M}_{s}$. Напомним, что $Z_{n_{i}}$ обозначает центральный многочлен алгебры общих матрищ порядка $n_{i}$, определенный в теореме 1.5 , где $n_{i}$ - размер $i$-го сомножителя. Выпишем многочлен $f$, задающий экстремальный $T$-идеал:

$$
f=\prod_{i=1}^{s}\left[Z_{n_{i}^{2}}, y_{i}\right]^{\varepsilon_{i}} C_{n_{i}^{2}}^{r}\left[Z_{n_{i}^{2}}, y_{i}^{\prime}\right]^{\delta_{i}},
$$

где $r$ равно индексу нильпотентности радикала, а величины $\delta_{i}$ и $\varepsilon_{i}$, соответствующие $i$-й клетке, принимают значение 0 или 1 и определяются по следуюшему правилу:

1) $\varepsilon_{i}=0$, если $i=1$ либо $n_{i} \leqslant n_{i+1}$;

2) $\delta_{i}=0$, если $i=s$ либо $n_{i}<n_{i+1}$;

3) в остальных случаях величины $\delta_{i}$ и $\varepsilon_{i}$ равны 1 ,

т.е. приоритет имеет клетка бо́льшего размера, а когда размеры совпадают, то правая.

При задании тождеств под произведением многочленов понимается такое произведение, когда в них подставлены непересекающиеся множества переменных. Например, $x^{r}$ есть $x_{1} \cdots x_{r}$, а

$$
x^{r}[z, t] x^{s}=x_{1} \cdots x_{r}[z, t] \cdot x_{r+1} \cdots x_{r+s} .
$$

Отметим, что множитель $\left[Z_{n}, y\right]$ не может располагаться на одной клетке размеpa $n$ и любая его специализация либо содержит клетки бо́льшего размера, либо радикальный элемент. Его роль - вынудить межклеточный переход. Множитель $C_{n^{2}}^{r}$ либо содержит клетку размера не меньшего $n$ (причем одно из $C_{n_{i}^{2}}$ входит туда полностью), либо принадлежит $r$-й степени радикала, что при больших $r$ ведет к равенству нулю. Таким образом, если $f \neq 0$, то все специализации для "множителя Капелли" отвечают $i$-й клетке. Подстановки в этот множитель дают возможность умножать на следы (формы), отвечающие этой клетке (см. п. 1.2). Дадим точную формулировку.

ПрЕДЛОЖЕНИЕ 2.1. а) Многочлен $f$ не является тождеством А.

б) При подстановке матричных единич из $\widehat{A}$, результат которой ненулевой, в каждое $C_{n_{i}^{r}}$ должны подставляться матричнье единицы, отвечающие $i$-й клетке.

в) $T$-идеал $T(f)$ является нётеровыц $R_{1} \otimes \cdots \otimes R_{s}$-модулем, где $R_{i}-$ кольцо следов (форм), отвечающих $i$-й клетке. Его размерность ГельфандаКириллова равна $\sum_{i} \operatorname{GKdim}\left(R_{i}\right)$.

ДокАЗАТЕЛЬСтво. Пункт а) очевиден, п. в) легко следует из п. б) и предложения 1.6, поскольку тензорное произведение нётеровых модулей (колец) нётерово. Поэтому остается доказать п. б).

Пусть $n=\max \left(n_{i}\right)$. Возьмем произвольную подстановку, при которой вместо каждой переменной подставляются матричные единицы. Рассмотрим множители $\left[Z_{n_{i}}, y\right]$ такие, что $n_{i}=n$. Каждый из них обрашается в нуль на соответствующей алгебре $\mathbb{M}_{n_{i}}$, так что в одну из переменных должна быть подставлена радикальная компонента алгебры и значение каждого должно лежать в ра- 
дикале. С другой стороны, поскольку $r$ равен индексу нильпотентности радикала, все сомножители $C_{n_{i}}$ в $C_{n_{i}^{r}}$ не могут лежать в радикале, и хотя бы один из них принимает полупростое значение. В силу максимальности $n_{i}$ оно должно соответствовать клетке размера $n=n_{i}$. Таким образом, каждому множителю $\left[Z_{n_{i}^{2}}, y\right]^{\varepsilon_{i}} C_{n_{i}^{2}}^{r}\left[Z_{n_{i}^{2}}, y\right]^{\delta_{i}}$ такому, что $n_{i}=n$, отвечает участок произведения, содержащий и радикальный множитель, и множитель, отвечающий полупростой клетке максимального размера. Поэтому все клетки максимального размера будут израсходованы. Кроме того, отметим, что $\varepsilon_{i}$ и $\delta_{i}$, соответствующие границам групп клеток размера $n$, равны 1 . Отсюда видно, что все клетки максимального размера будут заняты и все граничные операторы “входа-выхода-перехода", отвечающие их границам, тоже будут "истрачены".

Теперь возьмем $n^{\prime}=\max _{n_{i} \neq n}\left(n_{i}\right)$ и проведем для клеток порядка $n^{\prime}$ аналогичные рассмотрения. Далее доказательство завершается по индукции.

ЗАМЕчАниЕ. Степень $r$ при множителе необходима для данной конструкции. Может случиться так, что каждьй множитель вида $\left[Z_{n}, x\right]$ разместится в наборе "мелких клеток", а любое произведение вида

$$
\prod_{n_{i}<n}\left[Z_{n_{i}^{2}}, y\right]^{\varepsilon_{i}} C_{n_{i}^{2}}^{r}\left[Z_{n_{i}^{2}}, y\right]^{\delta_{i}}
$$

прекрасно размещается в одной клетке размера $n$.

Экстремальньй идеал для полупрямых произведений можно строить и методом Кемера и получить результаты, аналогичные только что изложенным.

Из наших рассмотрений также получается следуюшее

ПРЕДЛОЖЕНИЕ 2.2. Различные полупрямые произведения порождают различные многообразия. Пусть $\left\{A_{i}\right\}$ - неизоморфные полупрямые произведения, отличающиеся порядком сомножстелей. Тогда существуют многочленьи $\left\{f_{i}\right\}$ такие, что $\left.f_{i}\right|_{A_{i}} \neq 0$,но $\left.f_{i}\right|_{A_{j}}=0$ при $i \neq j$.

ЗАмЕчАниЕ. Вместо множителей $\left[Z_{n_{i}^{2}}, y\right]$, “вынуждающих межклеточные переходы", для той же цели можно взять полиномы, являющиеся тождествами $\mathbb{M}_{\max \left(n_{i}, n_{i+1}\right)}$ и не обращаюшиеся в нуль на $\mathbb{M}_{n_{i}} \rtimes \mathbb{M}_{n_{i+1}}$.

Итак, из предложения 2.1 вытекает нижняя оценка для размерности Гельфанда-Кириллова для полупрямых произведений. Она совпадает с нижней оценкой, полученной в предыдушем пункте и, таким образом, теорема 0.1 для многообразий, порожденных полупрямыми произведениями, доказана.

\section{§ 3. Редукция представимого случая к полупрямым произведениям}

Настоящий параграфф посвящен сведению случая относительно свободных представимых алгебр к полупрямым произведениям матричных алгебр. Полупрямые произведения матричных алгебр суть минимальные конечномерные алгебры, не содержащие идеалов с нулевым пересечением с данной полупростой частью (и с данньпм сложностным типом).

Teоpema 3.1 (A. Giambruno, М. Зайцев). Пусть $A$ - конечномерная алгебра над алгебраически замкнутыл полем $\mathbb{F}$ нулевой характеристики. Пусть 
$A=\bar{A}+J(A)$ есть разложсение $A$ в сумму полупростой части и радикала Джсекобсона. Пусть $\bar{A}=A_{1} \oplus \cdots \oplus A_{t}$, әде $A_{i} \simeq M_{d_{i}}(\mathbb{F})-$ простье компоненты $\bar{A}$. Если $A_{1} u_{1} A_{2} u_{2} \cdots u_{m_{1}} A_{m} \neq 0$ для некоторого $m \leqslant t u$ әлементов $u_{1}, \ldots, u_{m-1}$ из $J(A)$, то $A$ содержит подалгебру, изоморфную $U\left(\mathbb{F}, d_{1}, \ldots, d_{m}\right)$.

Нам понадобится следующее обобщение этой теоремы на случай произвольных полей.

Теорема 3.2. Пусть $A$ - конечномерная алгебра над полем $\mathbb{F}$. Пусть $A=\bar{A}+J(A)$ есть разложсение $A$ в сумму полупростой части и радикала Джсекобсона. Пусть $\bar{A}=A_{1} \oplus \cdots \oplus A_{t}$ есть разложсение $\bar{A}$ на простые компоненты. Если $A_{1} u_{1} A_{2} u_{2} \cdots u_{m-1} A_{m} \neq 0$ для некоторого $m \leqslant t$ элементов $u_{1}, \ldots, u_{m-1}$ из $J(A)$, то $A$ содержст набор подалгебр, изоморфных факторам полупрямого произведения (второго рода) $A_{1} \rtimes \cdots \rtimes A_{m}$. При этом само полупрямое произведение $A_{1} \rtimes \cdots \rtimes A_{m}$ вкладывается в прямую сумму этих подалгебр.

ДокАЗАТЕЛьство. Возьмем элементы $h_{i}, h_{i}^{\prime} \in A_{i}$, не являющиеся делителями нуля в $A_{i}$. Тогда $A_{1} h_{1} u_{1} h_{2}^{\prime} A_{2} h_{2} u_{2} h_{2} \cdots h_{m-1} u_{m_{1}} h_{m}^{\prime} A_{m} \neq 0$. Положим $v_{i}=h_{i} u_{i} h_{i+1}^{\prime}, i=1, \ldots, m-1$, и рассмотрим подалгебру, порожденную алгебрами $A_{i}$ и элементами $v_{i}$. Она является фактором полупрямого произведения. Достаточно показать, что само полупрямое произведение вкладывается в сумму алгебр, которые таким образом можно построить. Пусть $a_{i k} \in A_{i}$ и

$$
\sum_{k} \alpha_{k} a_{1 k} v_{1} a_{2 k} v_{2} \cdots a_{m k}=0
$$

для любых $v_{i}$ вида $v_{i}=h_{i} u_{i} h_{i+1}^{\prime}$. Проверим, что тогда

$$
\sum_{k} \alpha_{k} a_{1 k} \otimes a_{2 k} \otimes \cdots \otimes a_{m k}=0
$$

в тензорном произведении $A_{1} \otimes_{\mathbb{F}} \cdots \otimes_{\mathbb{F}} A_{m}$. В этом случае

$$
\sum_{k} \alpha_{k} a_{1 k} c_{1} a_{2 k} c_{2} \cdots a_{m k}=0
$$

в алгебре $A_{1} \rtimes \cdots \rtimes A_{m}$.

Возьмем базис в пространстве $A_{1} \otimes_{\mathbb{F}} A_{1} \otimes_{\mathbb{F}} A_{2} \otimes_{\mathbb{F}} A_{2} \otimes_{\mathbb{F}} \cdots \otimes_{\mathbb{F}} A_{m} \otimes_{\mathbb{F}} A_{m}$, состояший из элементов вида $h_{1 i_{1}} \otimes h_{1 i_{2}}^{\prime} \otimes \cdots \otimes h_{m i_{2 m}}^{\prime}$. Сопоставим каждому такому базисному вектору набор элементов $v_{i}=h_{i} u_{i} h_{i+1}^{\prime}$ и, соответственно, фактор полупрямого произведения. Достаточно показать, что если элемент $x=\sum_{k} \alpha_{k} a_{1 k} c_{1} a_{2 k} c_{2} \cdots c_{k} a_{m k}$ обрашается в нуль при любом таком морфизме, то ему соответствует нулевой элемент в тензорном произведении.

Расширив основное поле, ситуацию можно свести к случаю, когда $A_{i}$ суть матричные алгебры, в которых выбраны базисы из матричных единиц. Но тогда любой набор операторов перехода $\left\{E_{i j}^{k}\right\}$, где $i$ - индекс строки в $A_{k}, j$ - столбца в $A_{k+1}$, может быть реализован набором $\left\{v_{i}\right\}$. Поэтому если

$$
\sum_{k} \alpha_{k} a_{1 k} \otimes a_{2 k} \otimes \cdots \otimes a_{m k} \neq 0,
$$


то можно указать такие $E_{p q}, E_{r s}$ и $\left\{E_{i j}^{k}\right\}$, что одно слагаемое суммы

$$
\sum_{k} \alpha_{k} E_{p q} a_{1 k} E_{i_{1} j_{1}}^{1} a_{2 k} \cdots E_{i_{m} j_{m}}^{m} a_{m k} E_{r s}
$$

- не нуль, а остальные - нули. Но тогда и

$$
\sum_{k} \alpha_{k} a_{1 k} v_{1} a_{2 k} v_{2} \cdots a_{m k} \neq 0
$$

при $v_{k}=E_{i_{k} j_{k}}^{k}$, что противоречит выбору элемента $x$.

Пусть $A$ - относительно свободная $s$-порожденная представимая алгебра, $\mathfrak{M}=$ $\operatorname{Var}(A), A^{(i)}$ - ее первичные пирсовские компоненты, $n_{i}$ - сложность $A^{(i)}, k_{i}=$ $(s-1) \cdot n_{i}^{2}+1$, если $A^{(i)}$ бесконечна, и $k_{i}=0$, если $A^{(i)}$ конечна. Пусть $J(A)$ - радикал Джекобсона алгебры $A$ (он совпадает с нильрадикалом). Рассмотрим всевозможные ненулевые произведения $A(I)=A^{\left(i_{1}\right)} j_{1} A^{\left(i_{2}\right)} j_{2} \cdots j_{r_{I}-1} A^{\left(i_{r_{I}}\right)} \neq 0$, $j_{\alpha} \in J(A), i_{\mu_{I}} \neq i_{\nu_{I}}$ при $\mu \neq \nu$. Пусть $K(I)=\sum_{\alpha=1}^{r_{I}} k_{i}$.

Из предложения 0.3 и рассуждений из п. 2.1 вытекает

ПРЕДЛОЖЕНИЕ 3.3. Тогда $\operatorname{GKdim}(A) \leqslant \max _{I}\left(K_{I}\right)$.

Из теоремы 3.2 вытекает, что $\mathfrak{M}$ содержит всеполупрямые произведения $\mathbb{M}_{n_{I, 1}} \rtimes$ $\mathbb{M}_{n_{I, 2}} \rtimes \cdots \rtimes \mathbb{M}_{n_{I, r_{i}}}$, и потому оценка снизу для $A$ совпадает с максимальной нижней оценкой для таких многообразий. А для многообразий, порожденных полупрямьми произведениями, теорема 0.1 уже установлена. Поэтому из предложения 3.3 следует, что полученные верхняя и нижняя оценки совпадают. Таким образом, для многообразий, порожденных представимыми алгебрами, теорема 0.1 также доказана.

Из совпадения верхней оценки (равной размерности пространства параметров) и нижней оценки (равной максимальной размерности Гельфанда-Кириллова экстремального $T$-идеала в полупрямых произведениях) вытекает

ПреДЛОЖЕНИЕ 3.4 (о расширении нильпотентами). Пусть $A$ - относительно свободная представимая алгебра из многообразия $\mathfrak{M}=\operatorname{Var}(A), A_{t_{1}, \ldots, t_{r}}-$ ее расширение в многообразии $\mathfrak{M}$ конечным набором $\left\{t_{i}\right\}_{i=1}^{r}$, порождающим нильпотентый идеал. Тогда $\operatorname{GKdim}(A)=\operatorname{GKdim}\left(A_{t_{1}, \ldots, t_{r}}\right)$.

\section{§4. Редукция к представимому случаю}

\section{1. Утончение альтернаторов.}

4.1.1. Процедура перекачки. Метод перекачки представляет собой комбинаторную конструкцию, параллельную классическому понятию алгебраичности. Он позволяет единообразно решать ряд вопросов, относяшихся к PI-теории. Основная комбинаторная идея этого метода такова.

ПреДЛОЖенИЕ 4.1. Рассмотрим игру. Дано $n$ куч предметов. Первый игрок может выбрать любые $m$ куч и каждую из них разложить на правую и левую части. Второй игрок правые части нетождественно переставляет. Тогда первый игрок может добиться того, чтобы все кучи, кроме $m-1$, содержали не более чем по $m-1$ предмету. 
ДокАЗАТЕЛЬСтво. УПорядочим кучи. Составим вектор, $i$-я координата которого есть количество предметов в $i$-й куче. Упорядочим их лексикографически. Покажем, что если первый игрок не может увеличить вектор, соответствуюший кучам, то расположение предметов в кучах искомое.

В самом деле, пусть имеется $m$ куч; $k_{1}, \ldots, k_{m}$ - соответствующие количества предметов. Пусть $\forall i k_{i} \geqslant m$. Положим $k_{i}=k_{i}^{\prime}+q_{i}, q_{i}=i, k_{i}^{\prime}=k_{i}-i$. Поскольку $k_{i} \geqslant m$, то $k_{i}^{\prime} \geqslant 0$. Остается применить предложение 4.2.

ПРЕДЛОЖЕНИЕ 4.2. Пусть $k_{i} \geqslant m$, положим $k_{i}=k_{i}^{\prime}+q_{i}$. Пусть $k_{i}^{\prime} \geqslant 0 u$ $q_{j}>q_{i}$ при $j>i$. Тогда для любой нетождественной перестановки $\sigma \in S_{m}$ вектор $\vec{k}_{\sigma}=\left(k_{1}^{\prime}+q_{\sigma(1)}, \ldots, k_{m}^{\prime}+q_{\sigma(m)}\right)$ лексикографически меньше вектора $\vec{k}=\left(k_{1}^{\prime}+q_{1}, \ldots, k_{m}^{\prime}+q_{m}\right)$.

ДокАЗАТЕЛЬСТво. Если $\sigma(1) \neq 1$, то $\sigma(1)>1$ и $k_{1}^{\prime}+q_{\sigma(1)}>k_{1}^{\prime}+q_{1}$. В этом случае $\vec{k}_{\sigma} \succ \vec{k}$. Если $\sigma(1)=1$, то мы имеем индукционный спуск от $m$ к $m-1$.

Из предложения 4.1 получается

ЛЕмма 4.3 (о перекачке). Пусть $A$ - PI-алгебра, в которой выполняется полилинейное тождество $f$ степени т. Пусть слово $W$ имеет вид:

$$
W=c_{0} v_{1} c_{1} \ldots v_{m} c_{m+1}
$$

где $c_{i}-$ буквы, не входящие в слова $v_{j}$. Тогда $W$ по модулю $T(f)$ можно представить в виде линейной комбинации слов вида

$$
W^{\prime}=c_{i_{0}} v_{1}^{\prime} c_{i_{1}} \ldots v_{m}^{\prime} c_{i_{m+1}},
$$

где с $c_{i}$ не входят в слова $v_{j}^{\prime}$ и не более чем $m-1$ слово $v_{i}^{\prime}$ имеет длину, большую чем $m-1$.

Смысл этой леммы заключается в том, что с помощью тождества почти все символы из "куч" $v_{i}$ можно собрать в $m-1$ кучу $v_{i}^{\prime}$.

Мы играем роль первого игрока, когда представляем слово $W$ в виде произведения $W_{0} \cdots W_{m+1}$, "разрезая" при этом слова $v_{i}$. Далее тождество перерабатывает $W_{0} \cdots W_{m+1}$ в сумму слов, где $W_{i}$ нетождественно переставлены. Второй игрок выбирает самый “неприятный” член.

Из теории представления симметрической группы [1], [3] вытекает

ПРЕДЛОЖЕНИЕ 4.4. Существуют такое $k$ и коэффициенты $\alpha_{\sigma}$, что для любого полинома $F\left(x_{1}, \ldots, x_{k}, y_{1}, \ldots, y_{r}\right)$, полилинейного по $x_{i}$, выполняется равенство

$$
\sum_{\sigma} \alpha_{\sigma} F\left(c_{1} v_{\sigma(1)} d_{1}, \ldots, c_{k} v_{\sigma(k)} d_{k}, y_{1}, \ldots, y_{r}\right)=0
$$

В любой конечно порожденной алгебре выполняется тождество Капелли некоторого порядка [15]. В этом случае для некоторого $k$ можно положить $\alpha_{\sigma}=(-1)^{\sigma}$.

Точно так же, как и лемма о перекачке, с использованием этого предложения доказывается 
ЛЕмма 4.5 (о разреженной перекачке [3]). Пусть $A$ - PI-алгебра, в которой при всех $F$, полилинейных по переменным $x_{i}$, выполняется равенство (8). Подставим вместо $x_{i}$ слова $v_{i}$. Тогда $F\left(v_{1}, \ldots, v_{m}, \vec{y}\right)$ линейно представи́м әлементами вида $F\left(v_{1}^{\prime}, \ldots, v_{m}^{\prime}, \vec{y}\right)$, где не более чем $k-1$ слово $v_{i}^{\prime}$ имеет длину, больиую чем $k-1$.

4.1.2. Утончение многочленов Кемера. Пара $(A, H)$ состоит из алгебры $A$ с фиксированным набором образующих и натурального $H \in \mathbb{N}$.

Пусть $D$ есть некоторая диаграмма, многочлен $f \in S(D) \cap A_{\mathfrak{M}}\left\langle\bigcup_{i} \Lambda_{i}\right\rangle, g$ получается из $f$ путем подстановки слов $\left\{v_{j}\right\} \in A$ вместо переменных из $\bigcup_{i} \Lambda_{i}$. Если все эти слова имеют длину не выше $H$, то полином $g$ (и наборы $\Lambda_{i}$ ) называется тонким относительно $D$. (Поскольку это понятие относится к записи многочлена $g$, то требуется сушествование хотя бы одного такого представления.)

Если же хотя бы одно $v_{j}$ имеет длину больше $H$, то $g$ считается толстым. Это понятие относится не к самому многочлену $g$, а к его записи. (Мы допускаем такую вольность речи, когда ясно, какая запись рассматривается, и это не приводит к недоразумениям.)

Когда показатель $H$ не фиксирован, то можно говорить об $h$-тонких и $h$-толc$m b x$ многочленах для каждого $h \in \mathbb{N}$.

Если каждый многочлен Капелли толщины $h$ обращается в нуль, то будем говорить, что в алгебре выполняется система тождеств Капелли толщины $h$. Если $h=H$, то в алгебре выполняется система тонких тождеств Капелли. Аналогично определяются несколько более общие понятия выполнимости $c u c-$ темы разрехсенных тождеств толщины $h$, тонкой системы разрехенных тождеств, понятия толстого набора $\Lambda_{i}$ и набора $\Lambda_{i}$ толщины $h$, а также толщины переменной, рассматриваемой как набор из одного элемента.

Показатель $C H(v)$ есть длина слова $v$, если $f \in S(\Lambda), H(f)$ есть сумма длин слов, составляющих голову диаграммы $D$, a $T l(f)$-соответственно хвост.

$\mathrm{C}$ помошью процедуры перекачки непосредственно доказывается следуюшее

ПРЕДЛОЖЕНИЕ 4.6. Пусть в алгебре В выполняется система разрежсенных тождеств порядка $h$ и толщинь $h, g \in S(D)$. Тогда $g$ линейно представиім многочленами $g^{\prime} \in S(D)$ такими, что для каждого из них не более $h$ наборов переменных $\Lambda_{i}$ будут иметь толщину больше $h$. Более того, общее число переменных (во всех наборах) толщины больше $h$ не превосходит $h-1$.

СлЕДСТВИЕ 4.7. Если в алгебре В выполняется система тождеств Капелли порядка $m$ толщины $m$, то в ней выполняется также тождество Капелли порядка $2 m-1$.

Мы рассматриваем многочлены из $A_{\mathfrak{M}}\left\langle\bigcup_{i} \Lambda_{i}\right\rangle$ вместе с семейством соответствий мономов переменньм из набора $\bigcup_{i} \Lambda_{i}$, которое задает подстановку, результат которой есть $g$. Это позволяет говорить о moлcmы из $\bigcup_{i} \Lambda_{i}$, а также переменных толщины $h$.

ОПРЕДЕЛЕНИЕ 4.8. Через $S_{D, m_{1}, m_{2}, k}^{(m)}$ обозначим пространство, порожденное многочленами, отвечающими диаграмме $D \cup E$, и такими, что

i) диаграмма $E$ состоит из $m_{1}+m_{2}$ столбцов длины $k$;

ii) не более $m_{1}$ наборов переменных, отвечающих этим столбцам, имеют толшину больше $m$. 
Ясно, что $S_{D, m_{1}, m_{2}, k}^{(m)}$ является идеалом.

Нам понадобится еще одно следствие из предложения 4.6 и леммы о разреженной перекачке.

СледСТВИЕ 4.9 (утончение малых наборов). Пусть в алгебре выполняется система разрехсенных тохсдеств порядка $m$ толщины $m, m^{\prime}=m_{1}+m_{2} \geqslant m$. Тогда имеют место равенство

$$
S_{D, m^{\prime}, 0, k}^{(m)}=S_{D, m, m^{\prime}-m, k}^{(m)}
$$

и включение

$$
S_{D, m^{\prime}, 0, k}^{(m)} \subseteq S_{D, 0, m^{\prime}-m, k}^{(m)}
$$

Перейдем теперь к процедуре утончения больших наборов в многочленах Кемера. В основе лежит следуюшее соотношение. Пусть многочлен $f$ полилинеен и кососимметричен относительно набора переменных $\left\{y_{i}\right\}_{i=1}^{m}$ и линеен относительно переменной $x$. Рассмотрим многочлен

$$
f^{\prime}=f-\left.\sum_{i=1}^{m} f\right|_{x \mapsto y_{i} ; y_{i} \mapsto x} .
$$

Тогда многочлен $f^{\prime}$ полилинеен и кососимметричен относительно набора $\{x\} \cup$ $\left\{y_{i}\right\}_{i=1}^{m}$.

ЛЕмма 4.10. Пусть $m=|\Lambda|$, многочлен $f \in A_{\mathfrak{M}}\left\langle\Lambda \cup\left\{x_{0}\right\}\right\rangle$ полилинеен и кососимметричен относительно набора $\Lambda$ и линеен по переменной $x_{0}$. Пусть многочлен $g \in \mathrm{T}(f)$ получается путем подстановки вместо $x_{0}$ монома $v_{0}$ длинь $k, k>m+1$, а вместо переменных из $\Lambda$ - мономов длины не бо́льшей $m$.

Тогда $g$ линейно представим следующими многочленами:

1) значениями подстановок в $f$, для которых $C H\left(v_{0}^{\prime}\right)<C H(v)$;

2) многочленами, отвечающими подстановкам толщины $m+1$ әлементов А в многочлен из $A_{\mathfrak{M}}\left\langle\Lambda \cup\left\{x_{0}\right\}\right\rangle$, полилинейный и кососимметричный относительно набора $\Lambda \cup x_{0}$, и произвольных әлементов в другой набор $\Lambda^{\prime}$, где $\left|\Lambda^{\prime}\right|=m$.

ДокАЗАТЕЛьСТво. Выделим подмоном толшины $m+1$ монома $v_{0}$, обозначим его через $x_{0}^{\prime}$ и воспользуемся выражением (9). Остается заметить, что слагаемые, соответствующие

$$
f^{\prime}=f-\left.\sum_{i=1}^{m} f\right|_{x \mapsto y_{i} ; y_{i} \mapsto x}
$$

удовлетворяют условию 2), а слагаемые, соответствующие членам суммы

$$
\left.\sum_{i=1}^{m} f\right|_{x \mapsto y_{i} ; y_{i} \mapsto x},
$$

удовлетворяют условию 1) доказываемой леммы. В самом деле. Пусть $u$ есть подмоном монома $v$, причем $u$ включается в цепочку подмономов, возрастающих по включению, которая имеет максимальную длину. Тогда замена $u$ на моном ме́ньшей длины ведет к уменњшению показателя исходного монома.

Рассмотрим пару $(A, H)$. Пусть $H \geqslant k$. Аналогично доказывается 
ПРЕДЛОЖЕНИЕ 4.11. Пусть многочлен $f \in A_{\mathfrak{M}}\left\langle\bigcup_{i=0}^{1} \Lambda_{i}\right\rangle,\left|\Lambda_{0}\right|=k,\left|\Lambda_{1}\right|=$ $k+1$. Пусть набор $\Lambda_{1}$ содержст ровно s толстых переменных, а все переменные в наборе $\left|\Lambda_{0}\right|$ - тонкие. Тогда справедливы следующие утвержсдения.

а) Если $s>1$, то многочлен $g$, соответствующий $f$, линейно представи́м значениями многочленов $g_{\mu}$, отвечающих не более чем одной толстой переменной в наборе $\Lambda_{1}$.

б) Пусть $s=1$. Тогда многочлен $g$, соответствующий $f$, линейно представиім значениями многочленов $g_{\mu}$ таких, что показатели $(\mathrm{CH})$ всех переменных в наборе $\Lambda_{1}$ те же самые, а показатель одной переменной строго меньше.

(Все многочлень $g_{\mu}$ соответствуют $f$ при некоторой вербальной подстановке $x_{j} \mapsto v_{j}$, где $x_{j} \in \bigcup_{i=0}^{1} \Lambda_{i}$.)

ДокАЗАТЕЛЬСТво п. а) использует ту же конструкцию, связанную с формулой (9), п. б) есть следствие предыдущего утверждения, примененного к толстой переменной набора $\Lambda_{1}$.

Итак, мы утончаем большой набор ценой “утолшения” малого. Скомбинируем этот процесс с перекачкой, позволяющей утончать почти все малые наборы. Суммируя результаты предложения 4.11 и следствия 4.9, окончательно имеем

ПРЕДЛОЖЕНИЕ 4.12. Пусть $\mathfrak{M}$ есть многообразие алгебр с параметрами $(b, d, k)$, в котором выполняется разрехсенное тождество степени $m$, $S_{m_{1}, m_{2}}$ есть пространство значений многочленов Кемера с $m_{1}+m_{2}$ мальми столбиами, для которых все переменные, соответствующие больиим наборам, имеют толщину не более $m+1$, и не более чем $m_{1}$ переменных из мальх наборов имеют толщину свыше $m$ (а число больших наборов для многочлена Кемера всегда равно d). Тогда справедливь следующие утверәсдения.

а) Если $m_{1}=m-1$ и $m_{1}+m_{2} \geqslant k+1$, то пространство $S_{m_{1}, m_{2}}$ совпадает с пространством, порожденным всеми значениями полиномов Кемера с диаграммой $D$, состоящей из $d$ больших столбцов и $m_{1}+m_{2}$ мальх.

б) Пусть с есть число мальх столбиов в диаграмме Кемера D, диаграмма E состоит из т столбцов длинь $b$. Имеет место включение $H(D \cup E) \subset$ $S(0, c)$.

Смысл настоящего предложения (и цель данного параграфа) в мажорировании: пространство значений полиномов, отвечающих диаграмме Кемера $D \cup E$, содержится в пространстве тонких значений полиномов Кемера, отвечающих чуть ме́ньшей диаграмме $D$.

Суммируем результаты в удобном виде.

ПРЕДЛОЖЕНИЕ 4.13. Пусть $A$ - РІ-алгебра с показателем $(b, d, k)$, в которой выполняется система разреженных тождеств порядка $\mathrm{m}, A^{\prime}$ есть фактор по идеалу, порохсднному многочленами толщины $\max \{m, b+1\}$, отвечающим некоторой диаграмме Кемера для многообразия $\operatorname{Var}(A)$. Тогда пара $(b, d)$ для многообразия $\operatorname{Var}\left(A^{\prime}\right)$ строго меньие парьи $(b, d)$ для многообразия $\operatorname{Var}(A)$.

Итак, построен идеал $I$ ограниченной толщины, содержащий некоторьй $T$-идеал, фактор по которому имеет ме́ншшие численные характеристики. Из равенства 
$\operatorname{GKdim}\left(\bigoplus_{i=1}^{r} A_{i}\right)=\max \left(\operatorname{GKdim}\left(A_{i}\right)\right)$ следует, что $\operatorname{GKdim}(I)$ равна максимальной размерности Гельфанда-Кириллова пространства, отвечающего какой-то одной допустимой специализации элементов из множества $\left\{\Lambda_{i}\right\}$. Далее, из равенства $\operatorname{GKdim}(A)=\max \{\operatorname{GKdim}(I), \operatorname{GKdim}(A / I)\}$ следует, что верхние оценки на размерность Гельфанда-Кириллова относительно свободных ассоциативных алгебр оцениваются сверху размерностями Гельфанда-Кириллова представимых пространств типа $K_{\mathfrak{M}}(A, D)$.

4.2. Завершение доказательств. Теорема 0.1 для представимого случая уже установлена и наша цель - доказать

ПРЕДЛОЖЕНИЕ 4.14. Пусть для любого подмногообразия $\mathfrak{M}^{\prime} \subseteq \mathfrak{M}$, любой относительно свободной алгебры $A \in \mathfrak{M}$ и любой диаграммы Кемера $\Lambda$ размерность Гельфанда-Кириллова пространства $S_{\Lambda}(A)$ не превосходит размерности Гельфанда-Кириллова алгебры А. Тогда

$$
\operatorname{GKdim}(A)=\operatorname{GKdim}(A(\Lambda))=\operatorname{GKdim}(A / I),
$$

где I есть некоторый представимый идеал.

Поскольку нижние оценки (через сложностные характеристики) у алгебры и ее подходящего представимого фактора совпадают, нас интересуют только верхние оценки. Достаточно показать, что они совпадают с нижними оценками. В силу результатов п. 4.1 достаточно показать совпадение этих величин для пространств $K_{\mathfrak{M}}(A, D)$, которые представимы. Но предложение 3.4 утверждает совпадение размерностей Гельфанда-Кириллова этих пространств и полупрямых произведений. Это завершает доказательство теоремы 0.1 .

4.2.1. Алгебры обшего вида. Техника, связанная с представимьми пространствами и с утончением, переносится на случай алгебр обшей сигнатуры. Однако есть принципиальный момент, отличающий ассоциативный случай от общего. Для обеспечения индукционного спуска надо доказать, что размерность Гельфанда-Кириллова представимых пространств совпадает с размерностью исходной алгебры. Это не всегда вьполняется и размерность относительно свободной алгебры даже не обязательно целая [1], так что редукция к представимому случаю в этом месте не проходит. Чтобы редукция прошла, надо показать совпадение размерности Гельфанда-Кириллова $s$-порожденной алгебры общих элементов для $A_{0}(D)$ и размерности Гельфанда-Кириллова самой алгебры $A_{0}(D)$ (или, что то же самое, представимого пространства $K_{\mathfrak{M}}(A, D)$ ). Это обеспечивается, например, равенством базисного ранга многообразий $\operatorname{Var}(A)$ и $\operatorname{Var}\left(A_{0}(D)\right)$. Последнее условие можно ослабить (см. [1]): достаточно потребовать, чтобы любая относительно свободная алгебра из $\operatorname{Var}\left(A_{0}(D)\right)$ имела бы фактор с той же размерностью Гельфанда-Кириллова и базисньм рангом $s$ (в ассоциативном случае $s=2$ ). Это так, когда

1) размерность Гельфанда-Кириллова относительно свободной представимой алгебры определяется сложностным типом последней; сложностной тип многообразия $\mathfrak{M}$ определяется принадлежностью $\mathfrak{M}$ алгебр из некоторого класса $\mathscr{C}$

2) базисный ранг любого многообразия вида $\operatorname{Var}(C)$, где $C \in \mathscr{C}$, не превосходит $s$. 
В этом случае $\operatorname{GKdim}(A)$ при $r \geqslant s$ для относительно свободной $r$-порожденной алгебры $A \in \mathfrak{M}$ равна максимальной размерности Гельфанда-Кириллова своего фактора $A_{i}$ такого, что $\operatorname{Var}\left(A_{i}\right)=\operatorname{Var}\left(C_{i}\right)$, где $C_{i} \in \mathscr{C}$.

На самом деле настояшая работа посвяшена установлению того факта, что в ассоциативном случае в качестве класса $\mathscr{C}$ можно взять класс всех полупрямых произведений матричных алгебр с бесконечным центром.

\section{Список литературы}

1. Белов А.Я. Алгебры с полиномиальньми тождествами: представления и комбинаторнше методы // Дис. ... канд. докт. физ.-матем. наук. М.: МГУ, 2002.

2. Белов А.Я. О рациональности рядов Гилбберта относительно свободных алгебр // УМH. 1997. Т. 52. № 2. С. 153-154.

3. Belov A., Borisenko V., Latyshev V. Monomial algebras. New York: Plenum, 1998.

4. Procesi C. Rings with polynomial identities. New York: Marcel Dekker, Inc. VIII, 1973. (Pure Appl. Math. V. 17.)

5. Поликарпов С. В., Шестаков И. П. Неассоциативные афффинные алгебры // Алгебра и логика. 1990. Т. 29. №6. С. 709-703.

6. Поликарпов С. В. Свободные аффинные алгебры Алберта // Сиб. матем. журн. 1991. T. 32. №6 (190). C. 131-141.

7. Гришин А. В. Асимптотические свойства свободных конечно порожденных алгебр некоторых многообразий // Алгебра и логика. 1983. Т. 22. №6. С. 608-625.

8. Грииин А.В. Показатель роста многообразия алгебр и его приложения // Алгебра и логика. 1987. Т. 28. № 5. С. 536-557.

9. Giambruno A., Zaicev M. Minimal varieties of exponential growth // Proc. Amer. Math. Soc. 2001. V. 129. P. 59-67.

10. Giambruno A., Zaicev M. Minimal varieties of algebras of exponential growth // Adv. Math. 2003. V. 174. №2. P. 310-323.

11. Giambruno A., Zaicev M. Minimal varieties of algebras of exponential growth // Electron. Res. Announc. Amer. Math. Soc. 2000. V. 6. P. 40-44.

12. Джамбруно А., Зайщев М. В. Минималные многообразия с заданным ростом коразмерностей // Вестн. МГУ. Сер. 1. Матем., мех. 2003. Т. 1. С. 20-22.

13. Кемер А.Р. Тождества конечно порожденных алгебр над бесконечньм полем // Изв. АН СССР. Сер. матем. 1990. Т. 54. № 4. С. 726-753.

14. Зубрилин К. А. Алгебры, удовлетворяющие тождествам Капелли // Матем. сб. 1995. T. 186. №3. C. 53-64.

15. Кемер А.P. Нематричные многообразия, многообразия со степенньм ростом и конечно порожденные PI-алгебры // Дис. ... канд. физ. мат. наук. Новосибирск: НГУ, 1981.

Московский институт открытого образования,

Международный университет г. Бремена,

Поступила в редакцию

Hebrew University of Jerusalaem 